Revista Notas de Matemática

Vol.4(2), No. 268, 2008, pp.57-65

http://www.saber.ula.ve/notasdematematica/

Comisión de Publicaciones

Departamento de Matemáticas

Facultad de Ciencias

Universidad de Los Andes

\title{
Projective envelopes of finitely generated modules
}

\author{
Rafael Parra and Juan Rada
}

\begin{abstract}
Resumen
Un problema bien conocido en la teoría de envolturas es la caracterización de los anillos para los cuales los módulos (finitamente generados, finitamente presentados, simple) tienen una (pre)envoltura proyectiva. En estas notas introducimos los módulos $S$-proyectivos para una clase arbitraria $S$ de módulos finitamente generados, y caracterizamos de manera general los anillos para los cuales cada módulo en $S$ tiene una preenvoltura proyectiva.
\end{abstract}

Palabras Claves: Envolturas proyectivas, Módulos $f$-proyectivos, módulos simple - proyectivo, módulos finitamente generados.

\begin{abstract}
A well known problem in the theory of envelopes is to characterize those rings having the property that every (finitely generated, finitely presented, simple) module has a projective (pre)envelope. In these notes we introduce the $\mathcal{S}$-projective modules for an arbitrary class $\mathcal{S}$ of finitely generated modules, and characterize in this general setting those rings for which every module in $\mathcal{S}$ have a projective preenvelope.
\end{abstract}

key words. Projective envelopes, $f$-projective modules, simple-projective modules, finitely generated modules.

AMS(MOS) subject classifications. 16D10, 16D90

\section{Introduction}

Let $\mathcal{F}$ be a class of $R$-modules and $M$ an $R$-module. The concepts of $\mathcal{F}$-(pre)envelope and $\mathcal{F}$ (pre)cover of $M$ were introduced by Enochs [4]. The homomorphism $f: M \rightarrow F$, where $F \in \mathcal{F}$, is called a $\mathcal{F}$-preenvelope of $M$ if for every homomorphism $g: M \rightarrow F^{\prime}$, where $F^{\prime} \in \mathcal{F}$, there exists a homomorphism $h: F \rightarrow F^{\prime}$ such that $h f=g$. Furthermore, $f$ is called a $\mathcal{F}$-envelope of $M$ if every homomorphism $h: F \rightarrow F$ such that $h f=f$ is an automorphism. The $\mathcal{F}$-precover 
and $\mathcal{F}$-cover of $M$ are defined dually. The $\mathcal{F}$-envelope and $\mathcal{F}$-cover of a module if it exists it is unique.

A classical problem in this theory is the characterization of those rings having the property that every (finitely generated, finitely presented, simple) module has a (pre)envelope or a (pre)cover in a given class. In these notes we are concerned with projective (pre)envelopes of modules, i.e. $\mathcal{F}$-envelopes when $\mathcal{F}$ is the class of projective modules.

It is well known [3] that a ring $R$ is left coherent (resp. $\pi$-coherent) if and only if every finitely presented (resp. finitely generated) right $R$-module has a projective preenvelope. More recently, Mao [6] considered the problem of characterizing the rings for which every simple right $R$-module have a projective preenvelope; these turned out to be the right SPP rings, i.e. rings for which the left annihilator of every maximal right ideal is finitely generated.

In Section 2 we introduce for an arbitrary class $\mathcal{S}$ of finitely generated modules, the concept of $\mathcal{S}$-projective module. This is a natural generalization of the concepts $f$-projective [3] and the simple-projective [6] modules which played an important role in both papers. Following the results in [6] and having in mind the concept of locally initially small classes introduced in [7], we characterize the rings for which every module in $\mathcal{S}$ has a projective preenvelope. In particular, when $\mathcal{S}$ consists of the cyclic modules, we deduce that the rings $R$ for which every cyclic right $R$-module has a projective preenvelope are those whose left annihilator of every right ideal of $R$ is finitely generated.

\section{$2 \quad \mathcal{S}$-projective modules}

In this section, $\mathcal{S}$ will denote a class of finitely generated right $R$-modules.

Definition 2.1 A right $R$-module $M$ is $\mathcal{S}$-projective if for every homomorphism $f: S \rightarrow M$, where $S \in \mathcal{S}$, there exists homomorphisms $g: S \rightarrow L$ and $h: L \rightarrow M$, where $L$ is a free module, such that $f=h g$.

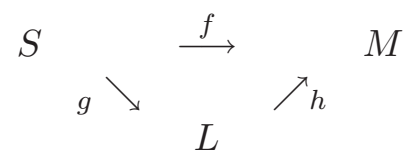

We denote by $\mathcal{S}$-proj the class of $\mathcal{S}$-projective modules.

Remark 2.2 Since $S$ is finitely generated, then if there is such a factorization then there is one through a finitely generated free module. 
Example 2.3 Every projective module is $\mathcal{S}$-projective. In fact, let $f: S \rightarrow P$ be a homomorphism, where $S \in \mathcal{S}$ and $P$ is projective. Then $P$ is a direct summand of a free module $L$, so there exists homomorphisms $u: P \rightarrow L$ and $v: L \rightarrow P$ such that $v \circ u=1_{P}$. Then

$$
v(u f)=1_{P} f=f
$$

and so $f$ factors through a free module.

Example 2.4 If $\mathcal{S}$ is the class of simple $\mathbb{Z}$-modules, then $\mathbb{Z}^{\mathbb{N}}$ is $\mathcal{S}$-projective but not projective.

Proposition 2.5 The class $\mathcal{S}$-proj is closed under direct summands.

Proof. Let $P$ be a direct summand of a $\mathcal{S}$-projective module $M$. Then there exists homomorphisms $u: P \rightarrow M$ and $v: M \rightarrow P$ such that $v u=1_{P}$. Let $f: S \rightarrow P$ be an homomorphism, where $S \in \mathcal{S}$. Since $M$ is $\mathcal{S}$-projective, there exists homomorphisms $g: S \rightarrow L$ and $h: L \rightarrow M$, where $L$ is free, such that $u f=h g$. It follows that

$$
v(h g)=v(u f)=1_{P} f=f
$$

and so $P$ is $\mathcal{S}$-projective.

Proposition 2.6 The class $\mathcal{S}$-proj is closed under pure submodules.

Proof. Let $Q$ be a pure submodule of a $\mathcal{S}$-projective module $N$. For every $S \in \mathcal{S}$ and homomorphism $f: S \rightarrow Q$, there exists a finitely generated free module $L$ and homomorphisms $g: S \rightarrow L$ and $h: L \rightarrow N$ such that $j f=h g$, where $j: Q \rightarrow N$ is the inclusion. Let $\phi: H \rightarrow N$ be a pure epimorphism, where $H$ is pure-projective. Consider the pullback diagram for $j$ and $\phi$ :

$$
\begin{array}{lllllllll}
0 & \rightarrow & K & \stackrel{\lambda}{\rightarrow} & H & \stackrel{\pi \phi}{\rightarrow} & N / Q & \rightarrow & 0 \\
& \downarrow^{\alpha} & & \downarrow^{\phi} & & \downarrow^{1} & & \\
0 & \rightarrow & Q & \stackrel{j}{\rightarrow} & N & \stackrel{\pi}{\rightarrow} & N / Q & \rightarrow & 0
\end{array}
$$

Since $L$ is projective, there exists a homomorphism $l: L \rightarrow H$ such that $h=\phi l$. Hence

$$
\pi \phi l g=\pi h g=\pi j f=0
$$

which implies $(l g)(S) \subseteq K$. Since $\lambda$ is a pure monomorphism we may consider $K$ as a pure submodule of $H$. Let $x_{1}, \ldots, x_{n}$ be a generator set for $S$. By [9], there exists a homomorphism 
$k: H \rightarrow K$ such that $k \lg \left(x_{i}\right)=\lg \left(x_{i}\right)$ for every $i=1, \ldots, n$. Consequently, $k \lg (x)=\lg (x)$ for every $x \in S$ and

$$
\begin{aligned}
\alpha k l g(x) & =j \alpha k l g(x)=\phi \lambda k l g(x)=\phi \lambda l g(x)=\phi \lg (x) \\
& =h g(x)=f(x)
\end{aligned}
$$

for every $x \in S$. Hence $\alpha k l g=f$ and so $Q$ is a $\mathcal{S}$-projective module.

Recall that a class $\mathcal{F}$ of right $R$-modules is locally initially small [7] if for every right $R$-module $M$ there exists a set $\mathcal{F}_{M} \subseteq \mathcal{F}$ such that every homomorphism $M \rightarrow F$, where $F \in \mathcal{F}$, factors through a direct product of modules in $\mathcal{F}_{M}$.

Corollary 2.7 The class $\mathcal{S}$-proj is locally initially small.

Proof. It follows from Proposition 2.6 and [7].

Let $A$ and $B$ be right $R$-modules and $B^{*}=\operatorname{Hom}_{R}(B, R)$ the dual module of $B$. We denote by $\Gamma_{A, B}$ the natural homomorphism $\Gamma_{A, B}: A \underset{R}{\otimes} B^{*} \rightarrow \operatorname{Hom}_{R}(B, A)$ which satisfies

$$
\Gamma_{A, B}(a \otimes g)(b)=a g(b)
$$

for all $a \in A, g \in B^{*}$ and $b \in B$.

The following result was proved for simples modules [6], however it can be extended to any class $\mathcal{S}$ of finitely generated modules.

Proposition 2.8 The following conditions are equivalent:

1. A is a $\mathcal{S}$-projective module;

2. $\Gamma_{A, B}$ is an epimorphism for every $B \in \mathcal{S}$.

Proof. 1. $\Rightarrow 2$. Let $B \in \mathcal{S}$ and $f \in \operatorname{Hom}_{R}(B, A)$. Since $A$ is a $\mathcal{S}$-projective module, there exists homomorphisms $g: B \rightarrow R^{n}$ and $h: R^{n} \rightarrow A$ such that $f=h g$. For each $i=1, \ldots, n$, let $\pi_{i}: R^{n} \rightarrow R$ and $\mu_{i}: R \rightarrow R^{n}$ be the canonical projections and injections. Set $a_{i}=h \mu_{i}(1)$ and $g_{i}=\pi_{i} g$. It follows easily from (1) that for every $b \in B$

$$
\Gamma_{A, B}\left(\sum_{i=1}^{n} a_{i} \otimes g_{i}\right)(b)=f(b)
$$


2. $\Rightarrow 1$. Let $B \in \mathcal{S}$ and $f \in \operatorname{Hom}_{R}(B, A)$. By hypothesis there exists $a_{i} \in A$ and $g_{i} \in B^{*}$ such that

$$
\Gamma_{A, B}\left(\sum_{i=1}^{n} a_{i} \otimes g_{i}\right)=f
$$

Define $g: B \rightarrow R^{n}$ by

$$
g(b)=\left(g_{1}(b), \ldots, g_{n}(b)\right)
$$

for every $b \in B$ and $h: R^{n} \rightarrow R$ by

$$
h\left(r_{1}, \ldots, r_{n}\right)=\sum_{i=1}^{n} a_{i} r_{i}
$$

Again by (1) it is easy to see that $h g=f$.

\section{Projective envelopes in a class of finitely generated modules}

Now we can state our main result.

Theorem 3.1 The following conditions are equivalent:

1. Every $S \in \mathcal{S}$ has a projective preenvelope;

2. Every $S \in \mathcal{S}$ has a free preenvelope;

3. Every $S \in \mathcal{S}$ has a $\mathcal{S}$-projective preenvelope;

4. Every $R$-module has a $\mathcal{S}$-projective preenvelope;

5. The class $\mathcal{S}$-proj is closed under direct products;

6. $R^{I}$ is a $\mathcal{S}$-projective module for every set $I$;

7. $S^{*}$ is finitely generated for every $S \in \mathcal{S}$.

Proof. 1. $\Leftrightarrow 2$. Let $S \in \mathcal{S}$ and $f: S \longrightarrow P$ a projective preenvelope. $P$ is a direct summand of a free module $L$, so there exists homomorphisms $u: P \longrightarrow L$ and $v: L \longrightarrow P$ such that $v u=1_{P}$. We will show that $u f$ is a free preenvelope. Let $g: S \longrightarrow L^{\prime}$ be a homomorphism, where $L^{\prime}$ is a free module

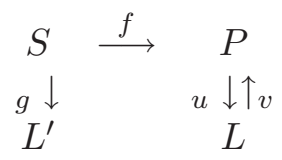


Since $f: S \longrightarrow P$ is a projective preenvelope, there exists a homomorphism $h: P \longrightarrow L^{\prime}$ such that $h f=g$. It follows then

$$
h v(u f)=h(v u) f=h f=g
$$

and so $u f$ is a free preenvelope.

2. $\Rightarrow 3$. Let $S \in \mathcal{S}$ and $f: S \rightarrow L$ a free preenvelope. Clearly, $L$ is $\mathcal{S}$-projective. Let $g: S \rightarrow M$ be a homomorphism such that $M$ is $\mathcal{S}$-projective.Then there exists homomorphisms $u: S \rightarrow K$ and $v: K \rightarrow M$, where $K$ is a free module and $g=v u$. Since $f$ is a free preenvelope, there exists a homomorphism $w: L \rightarrow K$ such that $w f=u$. Consequently, $(v w) f=v u=g$.

3. $\Rightarrow 2$. Let $S \in \mathcal{S}$ and $f: S \rightarrow M$ a $\mathcal{S}$-projective preenvelope. Then there exists homomorphisms $g: S \rightarrow L$ and $h: L \rightarrow M$, where $L$ is free, such that $f=g h$. We show that $g$ is a free preenvelope. Let $u: S \rightarrow L^{\prime}$ be a homomorphism where $L^{\prime}$ is free. Since $L^{\prime}$ is $\mathcal{S}$-projective and $f$ is a $\mathcal{S}$-projective preenvelope, there exists a homomorphism $v: M \rightarrow L^{\prime}$ such that $v f=u$. Then (vh) $g=v f=u$.

3. $\Rightarrow 5$. Let $\left\{M_{i}\right\}_{i \in I}$ be a family of $\mathcal{S}$-projective modules and $f: S \rightarrow \prod_{i \in I} M_{i}$ a homomorphism, where $S \in \mathcal{S}$. Consider the projections $\pi_{i}: \prod_{i \in I} M_{i} \rightarrow M_{i}$, for each $i \in I$. Let $g: S \rightarrow M$ be a $\mathcal{S}$-projective preenvelope of $S$. For each $i \in I$ there exists homomorphisms $g_{i}: M \rightarrow M_{i}$ such that $g_{i} g=\pi_{i} f$. On the other hand, since $M$ is $\mathcal{S}$-projective there exists homomorphisms $u: S \rightarrow L$ and $v: L \rightarrow M$, where $L$ is a free module, such that $g=v u$.

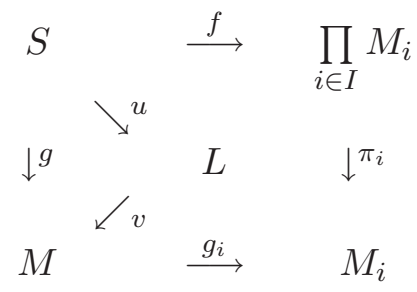

By the universal property of the direct product, there exists a (unique) homormophism $h: L \rightarrow$ $\prod_{i \in I} M_{i}$ such that $\pi_{i} h=g_{i} v$. Consequently, for each $i \in I$

$$
\pi_{i} h u=g_{i} v u=g_{i} g=\pi_{i} f
$$

and so $h u=f$. This shows that $\prod_{i \in I} M_{i}$ is $\mathcal{S}$-projective.

4. $\Longleftrightarrow 5$. By [7], every $R$-module has a $\mathcal{S}$-projective preenvelope if and only if the class $\mathcal{S}$-proj is locally initially small and $\operatorname{Summ}(\mathcal{S}$-proj) is closed under direct products. By Proposition 2.5 $\operatorname{Summ}(\mathcal{S}$-proj $)=\mathcal{S}$-proj and by Corollary $2.7, \mathcal{S}$-proj is locally initially small. 
4. $\Rightarrow 3$. This is clear.

5. $\Rightarrow 6$. This is clear because $R$ is $\mathcal{S}$-projective.

6. $\Rightarrow$ 7. Let $S \in \mathcal{S}$ and $I$ a set of indices. We have the following commutative diagram

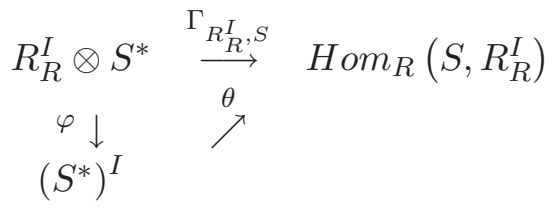

where $\theta$ and $\varphi$ are the canonical homomorphisms. Since $R^{I}$ is a $\mathcal{S}$-projective module, it follows from Proposition 2.8 that $\Gamma_{R_{R}^{I}, S}$ is an epimorphism, which implies that $\varphi=\theta^{-1} \Gamma_{R_{R}^{I}, S}$ is also an epimorphism. The result follows from [8, Lemma 13.1].

7. $\Rightarrow 1$. Let $S \in \mathcal{S}$ and $f_{1}, \ldots, f_{n}$ a set of generators of $S^{*}$. Define $f: S \longrightarrow R^{n}$ by

$$
f(x)=\left(f_{1}(x), \ldots, f_{n}(x)\right)^{\perp}
$$

We will show that $f$ is a projective preenvelope of $S$. Let $g: S \rightarrow P$ be a homomorphism where $P$ is projective. Since $P$ is $\mathcal{S}$-projective there exists homomorphisms $u: S \rightarrow R^{m}$ and $v: R^{m} \rightarrow P$ such that $g=v u$. For each $i=1, \ldots, m$, consider the projections $\pi_{i}: R^{m} \rightarrow R$. Then

$$
\pi_{i} u=r_{i 1} f_{1}+\cdots+r_{i n} f_{n}
$$

Define $\varphi: R^{n} \rightarrow R^{m}$ by

$$
\varphi=\left(\begin{array}{ccc}
r_{11} & \cdots & r_{1 n} \\
r_{21} & \cdots & r_{2 n} \\
\vdots & & \vdots \\
r_{m 1} & \cdots & r_{m n}
\end{array}\right)
$$

For each $x \in S$ and $i \in I$

$$
\begin{aligned}
\pi_{i} \varphi f(x) & =\pi_{i} \varphi\left(f_{1}(x), \ldots, f_{n}(x)\right)^{\perp} \\
& =\pi_{i}\left(\begin{array}{ccc}
r_{11} & \cdots & r_{1 n} \\
r_{21} & \cdots & r_{2 n} \\
\vdots & & \vdots \\
r_{m 1} & \cdots & r_{m n}
\end{array}\right)\left(f_{1}(x), \ldots, f_{n}(x)\right)^{\perp} \\
& =\pi_{i}\left(\begin{array}{c}
r_{11} f_{1}(x)+\cdots+r_{1 n} f_{n}(x) \\
\vdots \\
r_{m 1} f_{1}(x)+\cdots+r_{m n} f_{n}(x)
\end{array}\right) \\
& =r_{i} f_{1}(x)+\cdots+r_{i n} f_{n}(x)=\pi_{i} u(x)
\end{aligned}
$$


Hence, $\varphi f=u$. Finally,

$$
v \varphi f=v u=g
$$

which shows that $f$ is a projective preenvelope of $S$.

Theorem 3.1 unifies the results obtained by Ding and Chen [3], Mao [6], on the existence of projective preenvelopes of finitely generated, finitely presented or simple modules, respectively. Furthermore, in the case of cyclic modules we have the following result.

Corollary 3.2 The following conditions are equivalent:

1. Every cyclic right $R$-module has a projective preenvelope;

2. Every cyclic right $R$-module has a free preenvelope;

3. Every cyclic right $R$-module has a cyclic-projective preenvelope;

4. Every right $R$-module has a cyclic-projective preenvelope;

5. The class of cyclic-projective modules is closed under direct products;

6. $R_{R}^{I}$ is a cyclic-projective module for every set $I$;

7. $C^{*}$ is finitely generated for every cyclic right $R$-module $C$;

8. The left annhilator of every right ideal of $R$ is finitely generated.

Proof. Set $\mathcal{S}$ equal to the class of cyclic right $R$-modules in Theorem 3.1. $7 \Longleftrightarrow 8$ is a consequence of the fact that $C$ is cyclic if and only if $C \cong R / I$, where $I$ is a right ideal of $R$, together with the natural isomorphism $(R / I)^{*} \cong l_{R}(I)$, the left annhilator of $I$.

Remark 3.3 If $R$ is a semiperfect ring, then condition 1 in Theorem 3.1 can be replaced with "every $S \in \mathcal{S}$ has projective envelope". In fact, if $S^{*}$ is finitely generated for $S \in \mathcal{S}$, then since $R$ is semiperfect, there exists a projective cover $f: P \rightarrow S^{*}$, where $P$ is projective finitely generated. Then as in [2, Proposition 1], it can be shown that $S \stackrel{\sigma_{S}}{\rightarrow} S^{* *} \stackrel{f^{*}}{\rightarrow} P^{*}$ is a projective envelope of $S$.

\section{References}

[1] F.W. Anderson, K.R. Fuller, Rings and Categories of Modules, $2^{\text {nd }}$ Edition, Springer-Verlag, New York/Heidelberg/Berlin, (1992). 
[2] J. Asensio Mayor, J. Martinez Hernandez, Flat envelopes in commutative rings, Israel J. Math. 62 (1988) 123-128.

[3] N. Ding, J. Chen, Relative coherence and preenvelopes, Manuscripta Math. 81 (1993) 243262.

[4] E. Enochs, Injective and flat covers, envelopes and resolvents, Israel J. Math. 39 (1981) 189-209.

[5] E. Enochs, O. Jenda, Relative Homological Algebra. Berlin-New York: Walter de Gruyter, (2000).

[6] L. Mao, When does every simple module have a projective envelope? Comm. Algebra, 35 (2007) 1505-1516.

[7] J. Rada, M. Saorín, Rings characterized by (pre)envelopes and (pre)covers of their modules, Comm. Algebra 26 (1998) 899-912.

[8] B. Stenstrom, Rings of Quotients, Springer-Verlag, Berlin-New York, 1975.

[9] W. Zimmermann, On locally pure-injective modules. J. Pure Appl. Algebra 166 (2002) 337357.

\section{RAFAEL PARRA}

Departamento de Matemáticas, Facultad de Ciencias, Universidad de Los Andes

Mérida 5101, Venezuela

e-mail: rafaelparra@ula.ve

\section{JUAN RADA}

Departamento de Matemáticas, Facultad de Ciencias, Universidad Simón Bolívar

Caracas 1080, Venezuela

e-mail: juanrada@usb.ve 